flexion. The cervix was therefore completely divided by the hysterotome, the thick capsule of the tumour opened, and the tumour separated as far as the fingers could reach. The patient was so extremely anæmic that everything done to her was done in the constant fear of the production of heart-clot, and even the administration of ether in her case seemed risky. Nearly eight weeks elapsed after this preliminary proceeding before it was deemed safe to do anything more, when it was found that the opening into the capsule had closed, though the division of the cervix had remained permanent. On July 26 th the dilating plugs were applied, and next day the cervix was so open that a large free incision over the tumour was made, and the tumour freely separated. On August 4 th the tumour was found presenting through the os, and a large piece of it was removed by the écraseur. Un August 12 th the rest of it was found extruded and hanging by a thick pedicle, which was divided, and the mass removed. For many weeks after this operation she hung between life and death, and made a convalescence protracted over nearly eight months.

Mr. Tait is of opinion that in such a case removal of the ovaries is a much safer operation than enucleation of the tumour, and in his hands it has given much better results. Out of five cases where he has performed vaginal enncleation in the last three years there have been only two recoveries, whilst of seven similar cases in which he has performed oophorectomy there has been only one death, and that death was due solely to the operation having been too long delayed.

\section{SOUTH STAFFORDSHIRE GENERAL HOSPITAL.}

POPLITEAL ANEURISM ; TREATMENT BY FLEXION; CURE. (Under the care of Mr. NEWNHAM.)

GEORGE L-, aged forty-four, a goods guard, was admitted with popliteal aneurism. His previous history was excellent: he had never had syphilis, and had always been abstemious. Several months before he noticed the aneurism he wrenched his knee, and had to lay up for a short time. He could remember no other injury to the leg, nor could he recollect having made a forcible or sudden muscular effort to lift weights.

The history of the aneurism was as follows: Three weeks before admission (since Jan. 10th), the patient noticed a pulsation in the left popliteal space. His attention was directed to the seat of disease by pain in the calf when walking, the pain passing off if he stood still even for a moment. He thought that the swelling when he first noticed it was nearly as large as on a dmission.

On January 31 st he was taken in under the care of $\mathrm{Mr}$. Newnham. In the left popliteal space there was a tumour, about the size of a hen's egg. It had an extensile pulsation which could be arrested by pressure on the femoral artery. The area of pulsation extended over the popliteal space. There was a distinct bruit, and a thrill was communicated to the hand. On examining the other arteries of the body the left radial and temporal arteries were found feebler than the corresponding arteries on the opposite side, but no intrathoracic tumour could be detected. The superficial arteries had not an atheromatous feel, the heart was healthy, and no visceral disease could be discovered. The patient had a cataract of the right eye, which did not result from injury. He was a corpulent man, and somewhat aged in appearance, but his health, he said, was exceedingly good.

The patient was put on meat diet, and given five grains of the iodide of potassium and fifteen of the bicarbonate of potash. He was kept in bed and had an occasional purge. $\mathrm{On} \mathrm{Feb.} \mathrm{4th,} \mathrm{he} \mathrm{was} \mathrm{directed} \mathrm{to} \mathrm{keep} \mathrm{his} \mathrm{thigh} \mathrm{flexed} \mathrm{on} \mathrm{the}$ abdomen, and the leg on the thigh; the iodide was increased to ten grains. On the $6 \mathrm{th}$, he was put on a modified Bellingham's diet. Breakfast: bread and butter, two ounces; milk, two ounces. Dinner: meat, two ounces; bread, two ounces; beer, two ounces. Supper : bread and butter, two ounces; milk, two ounces. On the 8 th, the extensile pulsation had ceased in the tumour, although the pulsation in the popliteal and posterior tibial arteries could be felt. On the 9 th, the angle of flexion was increased; daily allowance of fluid fourteen ounces. On the 11th, flexion was discontinued; patient put on meat diet, with one pint of fluid. The aneurismal sac was felt to the inner side of the popliteal artery; it was a little larger than a walnut, and felt solid to the touch. The patient was left in bed for ten days after pulsation had ceased, and was discharged on the 9th of March, when the tumour could be scarcely felt.

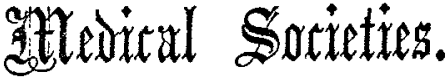

\section{ROYAL MEDICAL \& CHIRURGICAL SOCIETY.}

\section{Extra-uterine Gestation treated by Abdominal Section.-The} Treatment of Pelvic Suppuration by Abdominal Section and Drainage. - Sub-coracoid Dislocation of the Humerus.

THE ordinary meeting of this Society was held on the 11 th inst., J. E. Erichsen, Esq., F.R.S., President, in the chair. The subject of abdominal section was under discussion, introduced again by Mr. Lawson Tait, who, it will be remembered, contributed papers dealing with abdominal surgery earlier in the session. This time he related a third case of extra-uterine gestation, and in his second paper advocated the rather bold treatment of pelvic abscess by abdominal section. Both papers gave rise to some discussion. A paper by Mr. Eve, describing a rare variety of dislocation of the humerus, was also read.

A paper was read on "A Third Case of Extra-uterine Gestation, treated by Abdominal Section," by Mr. LAwsoN TAIT. The case differed from the two previously read before the Society in that the operation was performed before the death of the child, whose life was saved, but the mother succumbed to the operation. Mrs. Shad been married fourteen years, and had had six children. Since her last menstruation in May, 1879, she had been in constant pain, of which a severe attack occurred in July. In October she became satisfied she was pregnant, and Mr. Lattey, suspecting the nature of the case, asked Mr. Tait to see her. A large abdominal swelling was evidently a pregnancy, and to the left of the umbilicus was a prominent mass, which, from its rhythmic contraction, was clearly the uterus, and was attached to the larger mass behind it. The head of the child was detected between the rectum and the vagina. The pain becoming agonising, and being unrelieved by opiates, an operation was determined on. Ether was administered on the morning of Jan. 3lst, and on making the median incision the empty uterus was found, and the cyst, which was the right broad ligament, was opened, and the child removed without difficulty, the edges in the opening of the cyst being then secured to those of the parietal wound, leaving an opening for the discharge of the placenta, and the peritoneal cavity was closed. The child was at first profoundly nârcotised, but is now, three weeks after, thriving and well. 'The death of the mother, which occurred on the fourth day, seemed to be from protracted shock. At the post mortem there were found some morbid appearances in the liver and ante-mortem clots in the heart, but no peritonitis; the eyst proved to be the broad ligament as diagnosed, and there was a deciduous lining in the uterus in process of separation. The Fallopian tube had been ruptured at its lower aspects, the ovum thns escaping into the tissue of the broad ligament; and the case confirmed the author's opinion that all extra-uterine pregnancies are originally tubal, that the tube bursts in between eight and twelve weeks, and when not fatal an extra-uterine pregnancy is completed. The unfavourable result to the mother was due to the delay in the operation; but the case is of interest from the saving of the child. The child might have been removed by raginal section, but large venous sinuses in every direction made it likely that any injury to one of them would be fatal, and in vaginal section it is impossible to see what to avoid.-Mr. DORAN had recently dissected three cases of adranced tubal pregnancy, and had come to the conclusion that operating from the vagina was unjustifiable. The cyst in each of the three cases had pushed down the upper part of the rectum, which had become adherent to it. An incision of any length sufficient for the extraction of a fœtus could not have failed to wound the gut (and possibly a loop of small intestine as well pushed under the cyst); this would entail the most disastrous results. 